\title{
SOME SINGULAR PERTURBATION PROBLEMS
}

\author{
BY F. A. HOWES ${ }^{1}$
}

Communicated by Hans Weinberger, December 27, 1974

1. Introduction. The singularly perturbed boundary value problem

$$
\begin{gathered}
\epsilon y^{\prime \prime}=f\left(t, y, y^{\prime}, \epsilon\right), \quad 0<t<1, \\
y(0, \epsilon)=A, \quad y(1, \epsilon)=B,
\end{gathered}
$$

for $\epsilon$ a small positive parameter, has been studied extensively under various linearity restrictions. See, for example, [3] and [4] , and the references therein. However, two principal assumptions have been that the corresponding reduced problem

$$
\begin{gathered}
0=f\left(t, u, u^{\prime}, 0\right), \quad 0<t<1, \\
u(1)=B,
\end{gathered}
$$

has a solution $u=u(t)$ of class $C^{(2)}[0,1]$ and that in a suitable tube around $u, f_{y^{\prime}}=\partial f / \partial y^{\prime} \leqslant-k$, for some positive constant $k$. This latter assumption excludes the occurrence of turning points and makes the function $u$ a stable root of (1.3).

Under additional assumptions, by means of several asymptotic methods, the existence of a solution $y=y(t, \epsilon)$ of (1.1), (1.2), for each $\epsilon$ sufficiently small, can be deduced and this solution can be shown to satisfy an estimate of the form

$$
y(t, \epsilon)=u(t)+O\left(|A-u(0)| \exp \left[-k t \epsilon^{-1}\right]\right)+O(\epsilon), \quad 0 \leqslant t \leqslant 1 .
$$

Here $O$ denotes the standard Landau order symbol. The exponential term $v(t, \epsilon)=\exp \left[-k t \epsilon^{-1}\right]$ is a boundary layer function, in that $v(0, \epsilon)=1$ and $v(t, \epsilon) \rightarrow 0$ as $\epsilon \rightarrow 0^{+}$for $t>0$.

2. Statement of the problem and main result. Consider the more general boundary value problem

$$
a(t, \epsilon) y^{\prime \prime}=f\left(t, y, y^{\prime}, \epsilon\right), \quad 0<t<1,
$$

AMS (MOS) subject classifications (1970). Primary 34E15; Secondary 34B1 5.

${ }^{1}$ Supported by the National Science Foundation under Grant no. NSF-GP-37069X. 


$$
y(0, \epsilon)=A, \quad y(1, \epsilon)=B,
$$

and the corresponding reduced problem

$$
\begin{gathered}
a(t, 0) u^{\prime \prime}=f\left(t, u, u^{\prime}, 0\right), \quad 0<t<1, \\
u(1)=B,
\end{gathered}
$$

where $a(t, \epsilon)=a(t, 0)+\tilde{a}(t, \epsilon), \tilde{a}(t, \epsilon)>0$ and $\tilde{a}(t, \epsilon)=O(\epsilon)$, for $(t, \epsilon) \epsilon$ $[0,1] \times\left(0, \epsilon_{1}\right], \epsilon_{1}>0$.

Theorem. Assume (1) the problem (2.3), (2.4) has a solution $u=$ $u(t)$ of class $C^{(2)}(0,1) \cap C[0,1]$;

(2) the functions $f, f_{t}, f_{y}, f_{y^{\prime}}$ are continuous in $R: 0 \leqslant t \leqslant 1, \mid y-$ $u(t)|\leqslant d,| y^{\prime} \mid<\infty, 0 \leqslant \epsilon \leqslant \epsilon_{1}\left(d, \epsilon_{1}>0\right)$;

(3) there is a function $b=b(t, \epsilon)>0$, for $(t, \epsilon) \in[0,1] \times\left(0, \epsilon_{1}\right]$, such that $f_{y^{\prime}} \leqslant-b(t, \epsilon)$ in $R$;

(4) there is a constant $l>0$ such that $f_{y}\left(t, y, u^{\prime}(t), \epsilon\right) \geqslant l$ for $t \in$ $(0,1),|y-u(t)| \leqslant d$ and $0<\epsilon \leqslant \epsilon_{1}$;

(5) $\Gamma(a(t, \epsilon), b(t, \epsilon), \rho)=b^{2} a^{-1}\left(\rho-\rho^{2}\right)+a\left(b a^{-1}\right)^{\prime} \rho+l \geqslant 0$, for some constant $\rho>0$ and $(t, \epsilon) \in(0,1) \times\left(0, \epsilon_{1}\right]$;

(6) $\left|f\left(t, y, y^{\prime}, \epsilon\right)\right| \leqslant \phi\left(\left|y^{\prime}\right|\right)$, for $t \in[0,1],|y| \leqslant M,\left|y^{\prime}\right|<\infty$ and $0<\epsilon \leqslant \epsilon_{1}$, with $\phi$ positive, continuous and satisfying $\int^{\infty} s \phi^{-1}(s) d s=\infty$;

(7) $f\left(t, u(t), u^{\prime}(t), \epsilon\right)=f\left(t, u(t), u^{\prime}(t), 0\right)+\widetilde{f}(t, \epsilon)$, for $(t, \epsilon) \in(0,1)$ $\times\left(0, \epsilon_{1}\right]$;

(8) there is a function $\gamma=\gamma(t, \epsilon)$ such that $\gamma^{\prime} \leqslant 0$ and

(i) $a(t, \epsilon) \gamma^{\prime \prime}+b(t, \epsilon) \gamma^{\prime}-l \gamma \leqslant \widetilde{a}(t, \epsilon) u^{\prime \prime}(t)-\tilde{f}(t, \epsilon)$, for $(t, \epsilon) \in(0,1) \times\left(0, \epsilon_{1}\right]$;

(ii) $\gamma>0$ and $\gamma=O(\eta), \eta=\eta(\epsilon) \rightarrow 0$ as $\epsilon \rightarrow 0^{+}$, for $(t, \epsilon) \in$ $[0,1] \times\left(0, \epsilon_{1}\right]$.

Then for each $\epsilon, 0<\epsilon \leqslant \epsilon_{1}$, there exists a solution $y=y(t, \epsilon)$ of (2.1), (2.2). In addition,

$$
\begin{aligned}
y(t, \epsilon)=u(t)+o\left(|A-u(0)| \exp \left[-\rho \int_{0}^{t}\left(b a^{-1}\right)(s, \epsilon) d s\right]\right)+O(\eta), & \\
& 0 \leqslant t \leqslant 1 .
\end{aligned}
$$

The Theorem is proved by constructing Nagumo-type lower and upper solutions $\alpha, \beta$, respectively. See, for example, [2]. As an illustration, if $u(0) \geqslant A$, the functions

$$
\alpha(t, \epsilon)=u(t)-(u(0)-A) \exp \left[-\rho \int_{0}^{t}\left(b a^{-1}\right)(s, \epsilon) d s\right]-\gamma(t, \epsilon),
$$




$$
\beta(t, \epsilon)=u(t)+\gamma(t, \epsilon)
$$

satisfy the required inequalities.

3. Discussion. The Theorem includes a result like that mentioned in the Introduction, in that for $a(t, \epsilon) \equiv \epsilon$ and $b(t, \epsilon) \equiv k>0$,

$$
\Gamma(a(t, \epsilon), b(t, \epsilon), \rho=1) \equiv l>0 .
$$

Further, the assumptions that $u$ is of class $C^{(2)}[0,1]$ and $\tilde{f}=O(\epsilon)$ lead to the choice of $\gamma(t, \epsilon) \equiv \epsilon \sigma$, for $\sigma$ a sufficiently large positive constant.

A more interesting situation occurs when $a(t, \epsilon)=(t+\epsilon)^{2}$ and $b(t, \epsilon)=$ $k(t+\epsilon), k>0$. Then $\Gamma(a(t, \epsilon), b(t, \epsilon), \rho) \equiv 0$, provided $\rho=-r k^{-1}$, where $r$ is the negative root of the indicial equation $r(r-1)+k r-l=0$. If $u$ is of class $C^{(2)}[0,1]$ and $f=O(\epsilon)$, then again $\gamma \equiv \epsilon \sigma, \sigma \gg 1$, satisfies assumption (8). However, with this choice of $a$ and $b$, there can exist functions $u$ which only belong to $C^{(2)}(0,1) \cap C[0,1]$; as an example, consider the linear problem $(t+\epsilon)^{2} y^{\prime \prime}+2(t+\epsilon) y^{\prime}-y=0,0<t<1$. Then the function $\gamma$ is no longer of order $O(\epsilon)$; instead it satisfies $\gamma \rightarrow 0$ as $\epsilon \rightarrow 0^{+}$, for $t \in$ $[0,1]$, as follows from the computation in assumption (8). In addition, the boundary layer function is of algebraic type, for

$\exp \left[-\rho \int_{0}^{t}\left(b a^{-1}\right)(s, \epsilon) d s\right]=\exp \left[r k^{-1} \int_{0}^{t} k(s+\epsilon)^{-1} d s\right]=\left(1+t \epsilon^{-1}\right)^{r}$

Finally, the result of $\S 2$ can be applied to problems in which $f_{y}\left(t, y, u^{\prime}(t), \epsilon\right)$ is bounded and also problems in which $b(t, \epsilon)$ has a multiple character, for example, $b(t, \epsilon)=k+2 \epsilon\left(t+\epsilon^{2}\right)^{-1}$. Such functions $b(t, \epsilon)$ are briefly discussed in [1] with $a(t, \epsilon) \equiv \epsilon$.

\section{BIBLIOGRAPHY}

1. W. Eckhaus, Matched asymptotic expansions and singular perturbations, NorthHolland, Amsterdam, 1973.

2. L. K. Jackson, Subfunctions and second-order ordinary differential inequalities, Advances in Math. 2 (1968), 307-363. MR 37 \#5462.

3. R. E. O'Malley, Jr., Introduction to singular perturbations, Academic Press, New York, 1974.

4. W. R. Wasow, Asymptotic expansions for ordinary differential Equations: Trends and problems, Asymptotic Solutions of Differential Equations: Equations and Their Applications (Proc. Sympos., Math. Res. Center, U. S. Army, Univ. Wisconsin, Madison, Wis., 1964, Wiley, New York, 1964, pp. 3-26. MR 29 \#3724.

COURANT INSTITUTE OF MATHEMATICAL SCIENCES, NEW YORK,UNIVERSITY, NEW YORK, NEW YORK 10012 